\title{
Corneal Imaging and Densitometry Measurements in Patients with Fuchs' Dystrophy Undergoing Penetrating Keratoplasty and Descemet's Striping Automated Endothelial Keratoplasty
}

Khaled Alzahrani ( $\nabla$ khaledod@gmail.com )

The Universty of Manchester, Faculty of Biology, Medicine and Health https://orcid.org/0000-00032325-7688

\section{Arun Brahma}

Manchester Royal Eye Hospital

\section{Fiona Carley}

Manchester Royal Eye Hospital

\section{Chantal Hillarby}

The University of Manchester Division of Pharmacy and Optometry

\section{Research Article}

Keywords: ornea, Fuchs, Stroma, Densitometry, endothelial, dystrophy

Posted Date: April 20th, 2021

DOI: https://doi.org/10.21203/rs.3.rs-326343/v1

License: (c) (1) This work is licensed under a Creative Commons Attribution 4.0 International License. Read Full License 


\section{Abstract}

Aims:

In the present study, we used the densitometry software from the Oculus Pentacam to compare postoperative corneal clarity between penetrating keratoplasty (PK) and Descemet's stripping endothelial keratoplasty (DSAEK) in patients with Fuchs' dystrophy.

Methods:

A retrospective comparative study was carried out at Manchester Royal Eye Hospital. In 28 patients with Fuchs' dystrophy, corneal densitometry measurements were performed 12-18 months after corneal transplantation. The correlations of the densitometry measurements with the best corrected visual acuity (BCVA) and central corneal thickness (CCT) were analysed and compared between eyes that underwent PK and those that underwent DSAEK

Results:

Corneal densitometry measurements in the 33 eyes showed no significant differences between the PK and DSAEK post-surgery groups. There was no significant correlation between CCT and corneal densitometry measurements in either group ( $P>0.05$ in both cases). After DSAEK, corneal densitometry measurements were significantly correlated with BCVA in the central $(P=0.01)$, posterior $(P=0.007)$, and full-depth $(P=0.008) 0-2 \mathrm{~mm}$ zones of the cornea but not in PK group. The postoperative CCT was significantly different between the two groups (P凶0.01).

Conclusion:

The two types of corneal transplantation resulted in different outcomes in terms of corneal densitometry measurements. Improved visual acuity after DSAEK was found to positively correlate with improvements in corneal clarity. Oculus Pentacam provides an objective evaluation tool to monitor corneal status after surgery.

\section{Introduction}

Fuchs' endothelial dystrophy (FED) is usually treated using corneal transplantation [1, 2], which restores healthy endothelial function [3]. The first description of FED was published in 1900 by Ernst Fuchs, who described deposits known as guttata on the Descemet's membrane beneath the corneal endothelium; an abnormal extracellular matrix and endothelial cell loss were also apparent [4].

During the initial phase of the FED, epithelial oedema is absent. However, it builds up progressively in later stages. The degree of epithelial oedema is estimated by measuring the central corneal thickness (CCT). Corneal oedema begins within the central optical area and is a key cause of reduced vision in FED [5]. This is particularly severe in the morning due to reduced tear evaporation during sleep [6]. As the cornea 
becomes more oedematous, discomfort occurs, followed by extreme pain and photophobia. The eventual effects of extreme endothelial cell loss include stromal swelling, the creation of connective tissue in the stroma and epithelium, corneal oedema, and a clear vision reduction. \{Kim, 2004, Laser in situ keratomileusis versus laser-assisted subepithelial keratectomy for the correction of high myopia

In 1900, the first successful full-thickness keratoplasty was performed by Edward Zirm. Since then, this approach has seen considerable alteration and progress. Currently, over 3,000 corneal transplants are conducted annually in the United Kingdom alone [7]. FED transplants account for $25.8 \%$ of the overall proposed number for corneal transplant[8] .Historically, FED was treated using full-thickness (penetrating) keratoplasty (PK), which replaces all five corneal layers. However, as only the endothelial layer is diseased in FED, it is possible to use Descemet's stripping automated endothelial keratoplasty (DSAEK), whereby only the posterior stroma and endothelium are replaced [9].

The success of these surgical procedures is evaluated in terms of best-corrected visual acuity (BCVA) and CCT. Clinically, Pentacam (OCULUS Optikgerate $\mathrm{GmbH}$, Wetzlar, Germany) images are routinely used to assess patients undergoing transplant surgery. However, an update in computer software now allows corneal clarity to be measured in the same image. In the present study, we aimed to evaluate the use of corneal densitometry using Pentacam to assess the outcome of PK and DSAEK.

\section{Methods}

Participants:This study was a retrospective, comparative, non-randomised cross-sectional study. It was approved by the Central Manchester University Hospitals NHS Foundation Trust, Manchester, United Kingdom, and by the local national research ethics committee. The study followed the tenets of the Declaration of Helsinki, and written, informed consent was obtained from all subjects after the study's nature had been explained to them. The study included patients with FED who had been visiting the Cornea Clinic of the Manchester Royal Eye Hospital. Patients were recruited from the corneal transplantation database, which started on $3^{\text {rd }}$ of November 2015 till $1^{\text {st }}$ of December 2017 for adult participants and juvenile by $2^{\text {nd }}$ of June 2016 till December 2017.

Clinical notes were examined, and relevant information such as age, sex, date of surgery, and type of surgery was recorded for descriptive data analysis, as were postoperative parameters, including BCVA (in LogMAR) and clinical details.

Pentacam Imaging:All imaging was performed by the same trained staff in a dark room 12-16 months after surgery. A single image that met the quality requirements of Pentacam analysis was taken from each patient. This study's inclusion criteria were: age $\geq 18$ years, history of FED corneal transplantation (PK or DSAEK), completion of a 1-year post-treatment follow-up, availability of a Pentacam image from that time.

Surgical Procedures:The surgical procedures (DSAEK and PK) were each performed by one of two surgeons; they used identical techniques during the respective procedures to minimise variation [10]. PK 
was performed using a standard technique that employs a Hessburg-Barron trephine (JedMed Instrument Co., St. Louis, MO) [11]. DSAEK was conducted according to standard techniques [12-14]. As FED is a corneal disease associated with ageing, a triple procedure of cataract extraction, intraocular lens implantation, and corneal transplantation was performed in that order when necessary.

Statistical Analysis:Data analyses were carried out using SPSS V22.0 statistics software package for Windows. Descriptive statistics were presented as means \pm standard deviation. The data normality was examined using the Shapiro-Wilk test, which is more appropriate for small sample sizes $(\mathrm{N}<50)$. When parametric analysis was possible, the Student's t-test for two independent samples was used. All PValues less than 0.05 were considered statistically significant.

\section{Results}

A total of 33 eyes of 28 patients were studied. The sample characteristics were comparable between the groups; however, there were more women than men in both groups (PK, 11:2; DSAEK, 15:5); importantly, this did not constitute a significant difference between the groups $(P=0.96)$. The mean age of patients in the PK group was $70.0 \pm 10.0$ years, while that in the DSAEK group was $69.9 \pm 9.84$ years $(P=0.96)$.

In the PK group, seven eyes (53.8\%) achieved a postoperative BCVA of 0.3 LogMAR or better, while in the DSAEK group, 15 eyes (75\%) achieved a postoperative BCVA of 0.3 LogMAR or better. The mean postoperative BCVA in the DSAEK group was $0.19 \pm 0.12$ LogMAR, which was better than that in the PK group (0.37 \pm 0.22 LogMAR; $P=0.01)$.

Densitometry measurements showed that DSAEK causes less light scattering than PK in all layers of both the inner 0-2 mm zone of the cornea and the surrounding 2-6 mm annulus zone. However, further statistical analysis showed that none of the densitometry measurements' differences were significant (Pख0.05 in all cases; Table 1).

Table 1: Postoperative densitometry outcome in both central 0-2 $\mathrm{mm}$ and surrounding 2-6 $\mathrm{mm}$ of the corneal for anterior, middle, posterior and full depth layers for both PK and DSAEK. 


\begin{tabular}{|lllll|}
\hline Zones & Layers & PK $(M \pm S D)$ & DSAEK $(M \pm S D)$ & P Value \\
\hline 0-2 $\mathrm{mm}$ & Anterior & $26.05 \pm 3.16$ & $25.26 \pm 5.33$ & 0.63 \\
\cline { 2 - 5 } & Central & $23.19 \pm 3.05$ & $21.59 \pm 3.43$ & 0.18 \\
\cline { 2 - 5 } & Posterior & $18.34 \pm 2.54$ & $17.87 \pm 4.26$ & 0.72 \\
\cline { 2 - 5 } & Full Depth & $22.53 \pm 2.75$ & $21.56 \pm 3.77$ & 0.43 \\
\hline \multirow{2}{*}{-6 mm } & Anterior & $29.13 \pm 4.34$ & $26.44 \pm 5.8$ & 0.16 \\
\cline { 2 - 5 } & Central & $26.03 \pm 5.15$ & $22.55 \pm 5.68$ & 0.08 \\
\cline { 2 - 5 } & Posterior & $20.06 \pm 3.8$ & $18.38 \pm 4.66$ & 0.28 \\
\cline { 2 - 5 } & Full Depth & $25.07 \pm 4.31$ & $22.44 \pm 5.01$ & 0.11 \\
\cline { 2 - 5 } & & & \\
\hline
\end{tabular}

The mean CCT 1 year after PK treatment was $510.8 \pm 54.9 \mu \mathrm{m}$, while that 1 year after DSAEK treatment was $639.55 \pm 59.0 \mu \mathrm{m}$. Both CCT and the thinnest corneal area thickness were significantly different between the PK and DSAEK groups (Pख0.05) (Table 2).

Table 2: Postoperative central corneal thickness and thinnest area in both PK and DSAEK.

\begin{tabular}{|llll|}
\hline & PK $(M \pm S D)$ & DSAEK $(M \pm S D)$ & P Value \\
\hline CCT & $510.8 \pm 54.9$ & $639.55 \pm 59.0$ & $\otimes 0.01^{*}$ \\
\hline Thinnest Area & $493.5 \pm 60.7$ & $605.2 \pm 53.5$ & $\otimes 0.01^{*}$ \\
\hline
\end{tabular}

Postoperative BCVA was not significantly correlated with the densitometry measurements and CCT after surgery in the PK group. However, in the DSAEK group, both correlations were significant (Pख0.05; correlation between postoperative BCVA and densitometry measurement at the full-depth $0-2 \mathrm{~mm}: \mathrm{r}=$ $0.57, \mathrm{P}=0.008$; at the middle $0-2 \mathrm{~mm}: \mathrm{r}=0.53, \mathrm{P}=0.01$; at the posterior $0-2 \mathrm{~mm}: \mathrm{r}=5.80, \mathrm{P}=0.007$ ). Neither group showed a significant correlation between CCT and densitometry measurements in any corneal layers and any of the zones $(P>0.05)$. Figures 1 shows a linear regression comparison between the two groups at those zones found a significant correlation in DSAEK.

\section{Discussion}

DSAEK is a comparatively new method for transplanting the endothelium from a donor cornea, and it is now the primary surgical treatment of FED $[10,15,16]$. DSAEK has several advantages over PK in patients with endothelial dystrophy; it is associated with fewer complications and a shorter recovery time $[10,17,18]$. Several studies have reported that PK and DSAEK differ in several areas [18], including rates 
of rejection and post-operation BCVA. Updates from Nanavaty have reported similar findings [17]. However, up to our knowledge, the present study was the first to find that postoperative densitometry measurements differed between DSAEK and PK in patients with FED, which show a significant correlation to BCVA in the DSAEK group.

Scheimpflug imaging has numerous applications in corneal assessment. Moreover, with the updated software, it may be possible to measure the amount of backscattered light in diverse cornea regions [19, 20]. In this regard, corneal transparency is attributable to complex mechanisms, including size, regularity, and collagen fibrils arrangement [21].

The present study established that 12-18 months after surgery, the mean corneal densitometry in FED patients was somewhat higher than the normative value quantified in a previously published agematched set $[22,23]$. Patients who undergo PK still receive sutures that must be extracted, while patients who undergo DSAEK do not. Overall, in patients with PK, the corneal graft/host intersection requires 1224 months of recovery and complete suture extraction. Moreover, as PK involves full-thickness grafting, it contradicts the eyes' structural and immunological integrity and can cause more significant harm than DSAEK. Indeed, PK causes greater damage to corneal tissue. The greater activation of keratocytes likely occurs as a reaction to trauma, leading to raised corneal densitometry measurements.

The average endothelial cell density loss per year after successful PK ranges from $7.8 \%$ between 3 and 5 years after surgery [24] to $4.2 \%$ between 5 and 10 years after surgery [25]. This is contrasted with a remarkable $50 \%$ loss of endothelial cells just 6 months after successful DSAEK [26]. Because the present study involved only a small sample and was retrospective, we had limited ability to research this matter. Therefore, future research must conduct a well-planned research that explores the function of the endothelium pump or alternative factors contributing to enhanced corneal transparency in FED following DSAEK.

The present research confirmed that postoperative BCVA is significantly better after DSAEK than after PK, probably because partial suture extraction is necessary during PK. Regarding postoperative BCVA measurement, comparisons between DSAEK and PK are best carried out after entire suture extraction; an additional review of each patient's clinical notes revealed that postoperative BCVA is improved after entire suture extraction. The present study found a considerable association between postoperative BCVA and densitometry quantification in the DSAEK group, corroborating previous studies [27, 28]. However, this correlation did not occur in the PK group. Positive correlations were also found in the central, posterior, and full depth layers.

We should clarify that we excluded patients from our data analysis if they experienced complications during or after surgery. DSAEK is renowned for providing swifter visual rehabilitation than PK. Nonetheless, this has to be confirmed through additional research employing signals that are more dependable than visual results (e.g. contrast susceptibility and higher order aberration HOA steps following procedures), as well as larger sample sizes. 
After surgery, visual acuity improvements can take several months or even years $[26,29,30]$. In this regard, visual acuity can be affected by the lamellar graft's thickness and the interfere optical quality of the graft/host interface [28,31,32]. However, there was no correlation between BCVA and corneal thickness in the current study compared to the previous studies [33].

Increased corneal densitometry was found in the stroma, the anterior cornea; overall, corneal thickness was also improved. Therefore, the changes detected using densitometry appeared to affect the whole stroma and consisted of more than simply an increase in light scatter as a result of sub-epithelial fibrosis or augmented scatter from the graft/host interface, although both components still can cause light scattering in post-DSAEK patients.\{Kim, 2004, Laser in situ keratomileusis versus laser-assisted subepithelial keratectomy for the correction of high myopia

A significant relationship between visual outcome and corneal densitometry was recently discovered after DSAEK [27], corroborating observations of the present study. To some extent, Koh et al. reported higher values than we did. However, their study did not clearly define which thickness and diameter the densitometry measurements were acquired from. For this reason, the measurements are not directly comparable with those of the present study.

Poor graft interface can lead to graft failure. This illustrates the importance of maintaining a pristine graft interface and avoiding wrinkles in the graft across the pupillary area. In this regard, greater curvature disparities between recipient and donor corneas may promote wrinkle development $[15,34]$.

After surgery, gradual improvements in visual acuity are associated with increases in optical aberrations and corneal backscatter, which signify that the cornea is steadily restored after corneal oedema, with an average total corneal breadth of about $700 \mathrm{~mm}$. In fact, reduced BCVA after DSAEK is sometimes believed to be caused by corneal backscatter (haze) $[33,35,36]$. However, backscatter cannot influence vision since it is not distributed around the retina. In contrast, forward scatter does alter vision, but it naturally causes glare rather than diminished visual acuity [37-40].

The present study found that the patients' average CCT was $510.8 \pm 54.9 \mu \mathrm{m} 1$ year after PK and 639.55 $\pm 59.0 \mu \mathrm{m} 1$ year after DSAEK. This constituted a significant difference $(P=0.0001)$ and is similar to the results of [41] who documented an average CCT of $535.0 \pm 45.0 \mu \mathrm{m} 6$ months after PK; this had increased to $580.0 \pm 59 \mu \mathrm{m} 5$ years later. Nonetheless, the present study found no statistical correlation between BCVA and CCT in either group. Furthermore, CCT varies greatly among normal eyes.

The present study results agree with those of Terry et al. with regards to DSAEK graft thickness. They stated the following: 'graft thickness may have a small effect on visual outcomes in the extremes of thickness, but not in the common range of $100 \mathrm{~mm}$ to $200 \mathrm{~mm}$ ', and 'donor thickness has a tenuous relationship with visual outcomes, accounting for only $5 \%$ of the variance in vision between patients, and should play a minimal role in surgical planning' [39]. 
The present study was limited by its relatively small sample size and the possibility that suture removal may have affected the outcome, especially with respect to BCVA.

In conclusion, the present study showed that corneal densitometry can be used to detect subtle changes that occur in the cornea after transplantation and to monitor recovery. Densitometry may also further our understanding of the cornea in both normal and diseased conditions.

\section{Declarations}

\section{Competing intrests}

No conflect of intrest to be disclore by all the authors

\section{Funding info}

No fund have been recived for this research

\section{Author contributions}

All authors have been collebrated in study design ,data collections, data analysis, review the munscript after wreetinby first author

\section{Data Availability}

Data have been keept at university of Manchester data base and it can be available upon request

\section{Animal Research (Ethics)}

Not applicable

\section{Consent to Participate (Ethics)}

Done for all particpants

Consent to Publish (Ethics)

done

\section{Acknowledgement}

The authors thank the Manchester Royal Eye Hospital (corneal clinic) for supporting patient's recruitments for this study. Supported by Armed Forces Medical Services, Ministry of Defence, Riyadh, Saudi Arabia

\section{References}


1. Kang PC et al (2005) Trends in the indications for penetrating keratoplasty, 1980-2001. Cornea 24(7):801-803

2. Weisenthal R, Streeten B (1997) Posterior membrane dystrophies Cornea 2:1063-1090

3. Dapena I, Ham L, Melles GR (2009) Endothelial keratoplasty: DSEK/DSAEK or DMEK-the thinner the better? current Opinion in Ophthalmology 20(4):299-307

4. Bruinsma M, Tong C, Melles G, What does the future hold for the treatment of Fuchs endothelial dystrophy; will 'keratoplasty'still be a valid procedure\&quest. Eye (2013) 27(10): p. 1115-1122

5. Eghrari AO, Gottsch JD, Fuchs' corneal dystrophy. 2010

6. Fuchs E (1910) Dystrophia epithelialis corneae. Albrecht von Graefes Archiv für Ophthalmologie 76(3):478-508

7. Tan DT et al (2012) Corneal transplantation. The Lancet 379(9827):1749-1761

8. Gaum L et al (2012) Tissue and corneal donation and transplantation in the UK. Br J Anaesth 108(suppl_1):i43-i47

9. Espandar L, Carlson AN, Lamellar Keratoplasty: A Literature Review. Journal of ophthalmology (2013) 2013

10. Keenan TD et al (2011) Trends in corneal graft surgery in the UK. Br J Ophthalmol 95(4):468-472

11. Lim L, Pesudovs K, Coster DJ (2000) Penetrating keratoplasty for keratoconus: visual outcome and success. Ophthalmology 107(6):1125-1131

12. Gorovoy M, Price F (2005) New technique transforms corneal transplantation. Cataract Refract Surg Today 11:55-58

13. Pramanik S, Goins K, Sutphin J (2006) Corneal endothelial transplantation: Descemet's stripping endothelial keratoplasty (DSEK). EyeRounds. org

14. Gorovoy MS (2006) Descemet-stripping automated endothelial keratoplasty. Cornea 25(8):886-889

15. Price MO et al (2011) Descemet's stripping endothelial keratoplasty: five-year graft survival and endothelial cell loss. Ophthalmology 118(4):725-729

16. Terry MA et al (2009) Endothelial keratoplasty for Fuchs' dystrophy with cataract: complications and clinical results with the new triple procedure. Ophthalmology 116(4):631-639

17. Nanavaty MA, Wang X, Shortt AJ, Endothelial keratoplasty versus penetrating keratoplasty for Fuchs endothelial dystrophy. Cochrane Database Syst Rev, 2014. 2

18. Nanavaty MA, Shortt AJ (2011) Endothelial keratoplasty versus penetrating keratoplasty for Fuchs endothelial dystrophy. The Cochrane Library

19. Lopes B, Ramos I, Ambrósio R Jr (2014) Corneal densitometry in keratoconus Cornea 33(12):12821286

20. Alnawaiseh $\mathrm{M}$ et al (2015) Changes in corneal transparency after cross-linking for progressive keratoconus: long-term follow-up. J Refract Surg 31(9):614-618

21. Maurice DM (1957) The structure and transparency of the cornea. J Physiol 136(2):263-286 
22. Dhubhghaill SN et al (2014) Normative Values for Corneal Densitometry Analysis by Scheimpflug Optical AssessmentCorneal Densitometry in the Normal Population. Invest Ophthalmol Vis Sci 55(1):162-168

23. Alzahrani $\mathrm{K}$ et al (2017) Corneal clarity measurements in healthy volunteers across different age groups: Observational study. Medicine 96(46):e8563

24. Bourne WM, Hodge DO, Nelson LR (1994) Corneal endothelium five years after transplantation. Am J Ophthalmol 118(2):185-196

25. Ing JJ et al (1998) Ten-year postoperative results of penetrating keratoplasty. Ophthalmology 105(10):1855-1865

26. Koenig SB et al (2007) Visual acuity, refractive error, and endothelial cell density six months after Descemet stripping and automated endothelial keratoplasty (DSAEK). Cornea 26(6):670-674

27. Koh S et al (2012) Quality of vision in eyes after selective lamellar keratoplasty. Cornea 31:S45-S49

28. Ivarsen A, Hjortdal J, Recipient corneal thickness and visual outcome after Descemet's stripping automated endothelial keratoplasty. British Journal of Ophthalmology, 2013: p. bjophthalmol-2013304042

29. Price MO, Price FW (2006) Descemet's stripping with endothelial keratoplasty: comparative outcomes with microkeratome-dissected and manually dissected donor tissue. Ophthalmology 113(11):19361942

30. Li JY et al (2012) Three-year visual acuity outcomes after Descemet's stripping automated endothelial keratoplasty. Ophthalmology 119(6):1126-1129

31. Neff KD, Biber JM, Holland EJ (2011) Comparison of central corneal graft thickness to visual acuity outcomes in endothelial keratoplasty. Cornea 30(4):388-391

32. Busin M et al (2013) Ultrathin descemet's stripping automated endothelial keratoplasty with the microkeratome double-pass technique: two-year outcomes. Ophthalmology 120(6):1186-1194

33. Mencucci R et al (2015) Descemet stripping automated endothelial keratoplasty in Fuchs' corneal endothelial dystrophy: anterior segment optical coherence tomography and in vivo confocal microscopy analysis. BMC Ophthalmol 15(1):99

34. Price MO et al (2009) Descemet's membrane endothelial keratoplasty: prospective multicenter study of visual and refractive outcomes and endothelial survival. Ophthalmology 116(12):2361-2368

35. Anshu A et al (2012) A cause of reticular interface haze and its management after Descemet stripping endothelial keratoplasty. Cornea 31(12):1365-1368

36. Hindman HB et al (2013) Post DSAEK optical changes: a comprehensive prospective analysis on the role of ocular wavefront aberrations, haze, and corneal thickness. Cornea 32(12):1567

37. Unterlauft JD et al (2015) Intraocular Pressure and Trabecular Meshwork Outflow Facility After Descemet Stripping Endothelial Keratoplasty. Journal of glaucoma

38. Terry MA et al (2009) Precut tissue for Descemet's stripping automated endothelial keratoplasty: vision, astigmatism, and endothelial survival. Ophthalmology 116(2):248-256 
39. Terry MA et al (2012) Descemet's stripping automated endothelial keratoplasty: the tenuous relationship between donor thickness and postoperative vision. Ophthalmology 119(10):1988-1996 40. Wacker K, Bourne WM, Patel SV (2016) Effect of Graft Thickness on Visual Acuity After Descemet Stripping Endothelial Keratoplasty: A Systematic Review and Meta-Analysis. Am J Ophthalmol 163:18-28

41. Verdier DD et al., Corneal thickness as a predictor of corneal transplant outcome. Cornea, 2013. 32(6)

\section{Figures}

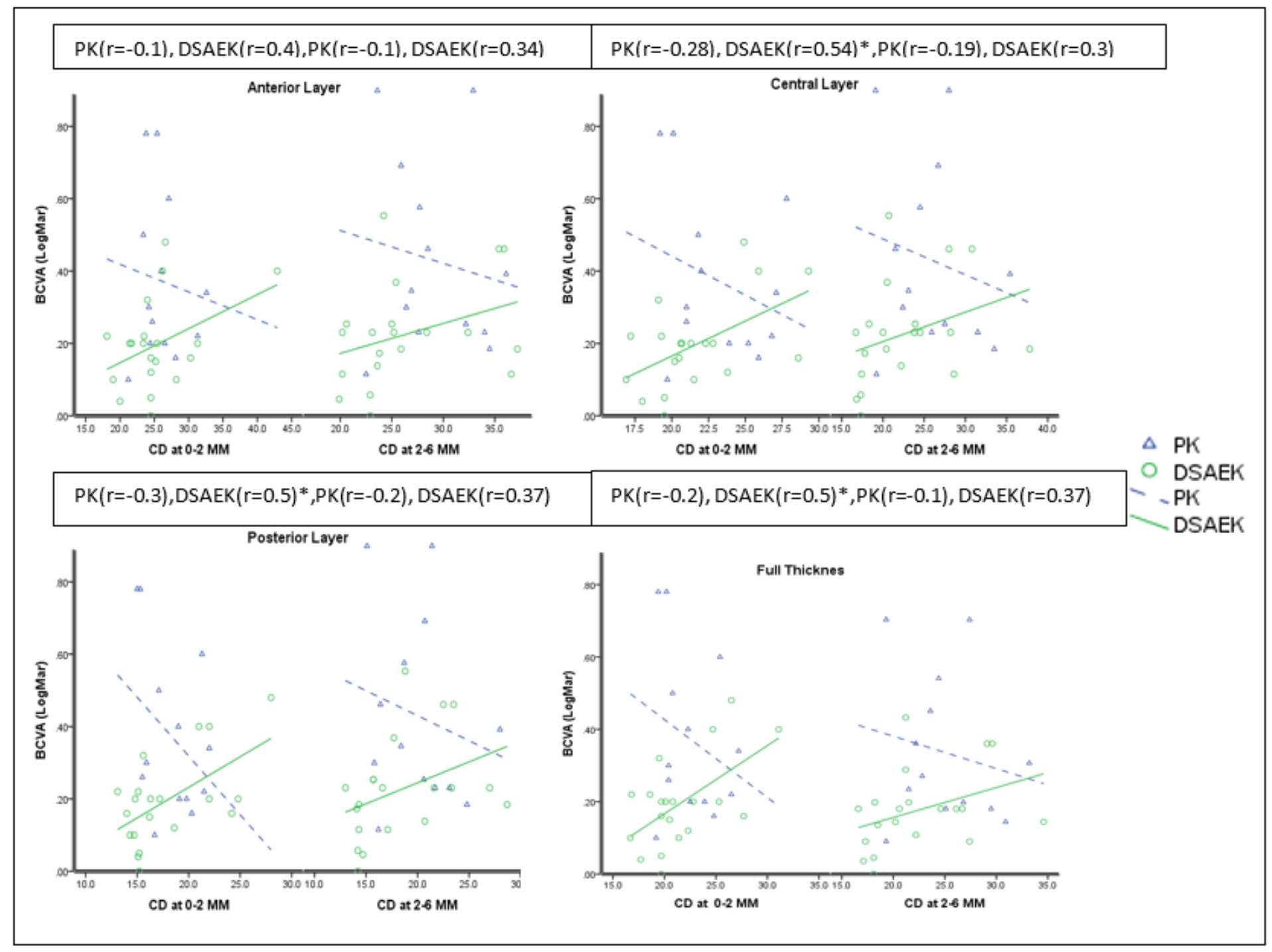

\section{Figure 1}

The correlation between BCVA and Corneal densitometry in PK and DSAEK (* represent significant at Pvalue of $<0.05)$. 